\title{
INTERACTION OF STATE ESTIMATION AND SENSITIVTY ANALYSIS FOR THE OPERATION OF A REAL FLEXIBLE DISTRIBUTION GRID
}

\author{
Marco WAGLER \\ Professorship for Power Transmission Systems \\ Technical University of Munich (TUM) - Germany \\ marco.wagler@tum.de
}

\begin{abstract}
In the following paper a sensitivity analysis based on state estimation methods is presented. The analysis is performed for a real low voltage grid and the suitability for real-time applications is demonstrated. The term sensitivity hereby expresses the impact of a load flow modification in a particular node with respect to other node voltages, line currents and transformer loadings. The overall errors of the sensitivity values are investigated with the help of symmetrical simulations, as well as evaluations of unsymmetrical field test scenarios. Recommendations for a practical implementation within a flexible distribution grid are given.
\end{abstract}

\section{INTRODUCTION}

One way to reduce the requirement for distribution system reinforcement resulting from renewable energy integration is the application of a flexible distribution grid concept. The idea of flexible distribution grids is the activation of flexibilities (active $(\mathrm{P})$ or reactive $(\mathrm{Q})$ power modification) to avoid critical grid operation scenarios, such as line congestion or over and under voltages. A flexible distribution grid can offer its flexibilities on a day-ahead basis with the help of forecasts in order to prevent dangerous grid states from the outset. In addition to the day-ahead trading, the possibility for live system modifications allows a flexible distribution grid to react to forecast errors in real-time. For the successful operation of a flexible distribution grid, a sensitivity analysis is necessary to determine the most effective nodes that must be modified in order to correct the dangerous grid state. For the calculation of such a sensitivity analysis, all node voltages of the grid must be known, in addition to the bus admittance matrix. For day-ahead planning, the node voltages are gained with the help of standard load flow calculations. However, for live applications the node voltages must be gained with the help of a state estimation. This leads to unavoidable errors in the calculated node voltages depending on factors such as the number, location and accuracy of installed meters. Further, the sensitivity analysis is based on a linearization of the load flow problem (derived from the modified Newton-Raphson Jacobian matrix [1]), which introduces additional error. This paper investigates the error of the sensitivity values calculated for a real low voltage grid, both in simulation and in field-test trials [2]. The sensitivities are computed from node voltages

\author{
Rolf WITZMANN \\ Professorship for Power Transmission Systems \\ Technical University of Munich (TUM) - Germany \\ rolf.witzmann@tum.de
}

that are derived from a state estimation and so are potentially inaccurate. The mathematical derivation for the calculation of the sensitivity matrices is presented in [1]. The partial differentials for the node voltages $\frac{\delta|V|}{\delta P}$ and $\frac{\delta|V|}{\delta Q}$ as well as the line and transformer currents $\frac{\delta\left|I_{L}\right|}{\delta P}$, $\frac{\delta\left|I_{L}\right|}{\delta Q}, \frac{\delta\left|I_{T}\right|}{\delta P}$ and $\frac{\delta\left|I_{T}\right|}{\delta Q}$ are presented. It was shown that the sensitivity values exhibit errors caused by linearization. Nevertheless, most of the errors were within a range of $\pm 10 \%$ and even less in critical situations. This result was based on correct voltage values determined by design relevant load flow simulations. For the implementation of a live flexible distribution grid, the voltage values of all nodes are provided by a state estimation. The rural Bavarian low voltage grid under investigation consists of 71 nodes and is connected to the $20 \mathrm{kV}$ grid by a $630 \mathrm{kVA}$ transformer. The grid contains five PV systems, four of which are equipped with a controllable battery storage system. Furthermore household and industrial loads are connected to the grid. The state estimation is based on power quality analyzers measuring bus-voltages as well as $\mathrm{P}$ and $\mathrm{Q}$ branch flows. These PQ analyzers are installed in a number of power distribution cabinets and the transformer station. In addition, smart meters are installed at the households with battery storages, metering active power injections and the respective bus voltages.

\section{SIMULATION RESULTS}

To investigate the errors of the sensitivity values, a load flow calculation for a representative summer and winter week with a resolution of 15 minutes was performed within the scope of this paper. The simulation addresses the question of whether the node voltage inaccuracies resulting from the state estimation lead to an unacceptable worsening of the sensitivity values. It should be mentioned that all calculations were conducted symmetrically.

\section{$\underline{\text { State Estimation }}$}

For the state estimation, all load flow results that are used as measurements are superimposed with randomly distributed errors to simulate the limited accuracy of the meters used in the field-test. Hatchel's augmented matrix approach was used to compute all node voltage magnitudes and angles. This algorithm is an advancement of the common Weighted Least Square Method, resulting in a better numerical stability due to 
the separation of the virtual measurements [3]. A detailed mathematical derivation of Hatchel's augmented matrix approach can be found in [4] and [5]. Table 1 gives an overview of the number of measurements within the grid. Virtual measurements hereby represent zero active and reactive power injections, whilst pseudo measurements are load measurements which are not measured in reality and therefore enter the algorithm with a distinct lower weighting factor. However, they are required for observability reasons. Standard load profiles are therefore used, which are scaled to both the known yearly consumption and the actual transformer loading. In order to minimize the estimation error, more than $2 \mathrm{~N}$ 1 ( $\mathrm{N}=$ number of nodes) measurements are required which is quantified by the level of redundancy. Similar to [6], a redundancy level of 1.25 is achieved through the assumption of unrestricted functionality of all measurement devices in the simulations.

\begin{tabular}{|c|c|c|c|}
\hline Real & Virtual & Pseudo & Redundancy \\
\hline 40 & 90 & 46 & 1.25 \\
\hline
\end{tabular}

Table 1: Number of measurements within the grid
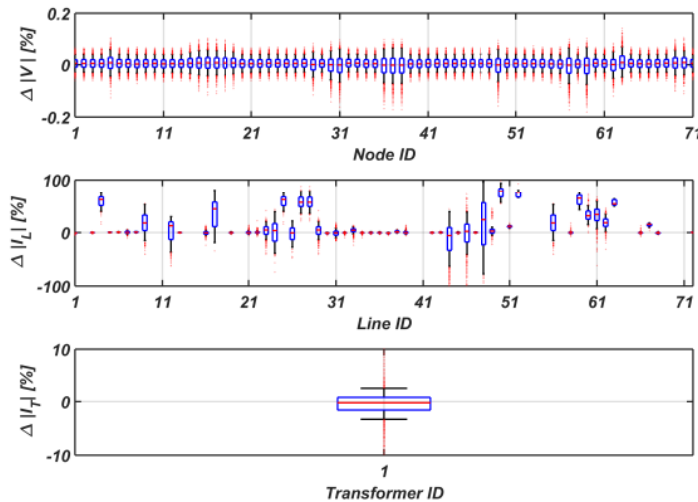

Figure 1: State Estimation error distribution for each node, line (only situations with $\left|\mathrm{I}_{\mathrm{L}}\right|>5 \mathrm{~A}$ ) and transformer in relation to the real value for the considered simulation period.

Figure 1 quantifies the distribution of the errors made by the state estimation relating to the real value represented by the results of the load flow simulations. The edges of the blue boxes describe the $25^{\text {th }}$ and the $75^{\text {th }}$ quantile, whereas the black borders describe the $5^{\text {th }}$ and the $95^{\text {th }}$ quantile. The red dots represent outliers. The median is displayed by a red line. It can be seen that no outlier is exceeds $0.2 \%$ for $\Delta|\mathrm{V}|$, implying a very accurate performance of the state estimation. Furthermore, the errors for the transformer loading are mostly within an acceptable range of $\pm 5 \%$. In contrast, the error distribution of the line currents shows lines with very accurate results as well as lines with estimation errors up to $100 \%$. The reason for this insufficient accuracy is the inaccurate estimation of the voltage angles at the respective points which are crucial for the current flow over the line. However all insufficient results were gained at house connection lines without measuring points. An overloading at these lines is very unrealistic and therefore the high inaccuracy does not pose a significant problem. All lines where a hazard of overloading is realistic exhibit an acceptable error in the range of $\pm 10 \%$. In the case of an inadequate evaluation by the respective distribution system operator, an improvement of the state estimation could be achieved through the installation of additional measurement devices.

\section{Sensitivity Analysis}

The lower part of figure 2 and 3 displays the active power sensitivity curve of a node $\mathrm{j}$ related to the voltage magnitude at node $\mathrm{i}$ and the line current magnitude at line $\mathrm{k}$ respectively, whereby neither node $\mathrm{i}$ nor line $\mathrm{k}$ were measured. The real sensitivity values were calculated by a step by step modification of active and reactive power at every node and a subsequent load flow simulation to observe the effects at every node.
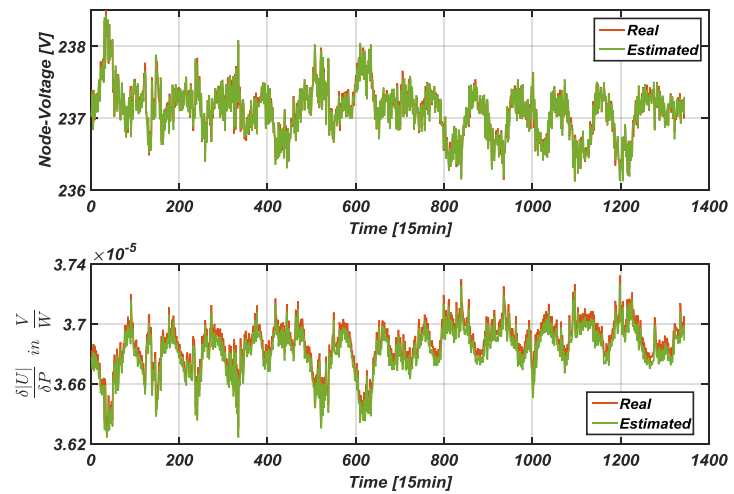

Figure 2: Node voltage at point $\mathrm{i}$ and representative sensitivity behavior from node $\mathrm{j}$ to $\mathrm{i}$ for real and estimated values for a simulation period of two weeks.
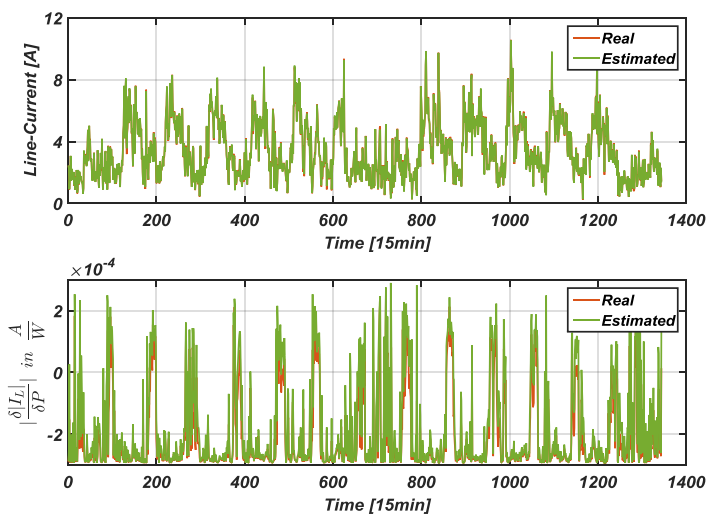

Figure 3: Line current at line $\mathrm{k}$ and representative sensitivity behavior from node $\mathrm{j}$ to $\mathrm{k}$ for real and estimated values for a simulation period of two weeks. 
Based on the estimated voltages and currents displayed in the upper section of each respective figure, it can be seen that in both cases the real sensitivity values are nearly congruent with calculated sensitivity values. In addition, the voltage sensitivity value does not change significantly over time. This circumstance is justified by the bigger dependence on the admittance matrix as on the actual voltage. However, the line current sensitivity features a highly fluctuating behavior which indicates a distinct dependence on the voltage values and the present load flow direction. Figure 4 shows the error distribution of all occurring sensitivity values within the simulation period. The sensitivity errors are calculated according to following exemplarily formula for $\mathrm{P}$ modification.

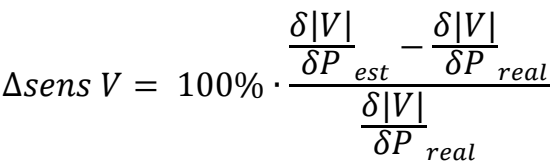
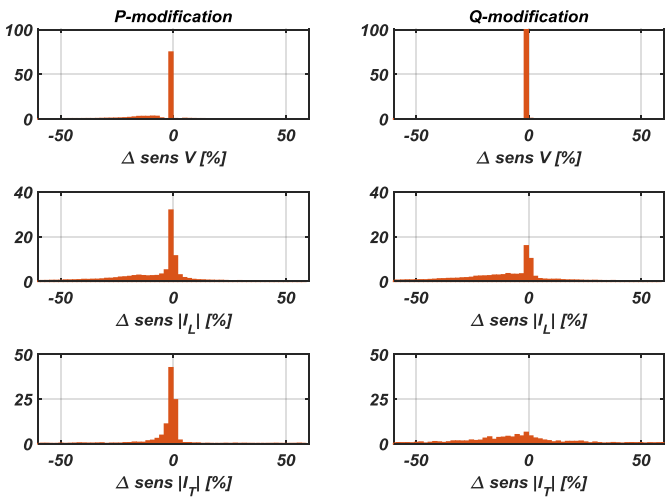

Figure 4: Overall sensitivity error distribution for a simulated period of two weeks in \% referring to the real value.

As expected, the voltage sensitivity values for both $\mathrm{P}$ and Q-modification show a high accordance between estimated and real sensitivity values. In addition, the line and transformer current sensitivity values display a very low mean error. However, the width of the distribution curve is increasing. The question arises whether these higher errors are still acceptable for the application of a live flexible distribution grid. To answer this question it is necessary to mention that only high sensitive values would be chosen to counteract a problem. It is therefore of the upmost importance that high sensitive values correlate with low errors. Figure 5 reveals the relation between sensitivity value and error for all $\frac{\delta\left|I_{L}\right|}{\delta P}$ and $\frac{\delta\left|I_{T}\right|}{\delta Q}$ calculations. It can clearly be seen that high sensitivity values correlate with a small error. Due to this, the error magnitude does not pose a constraint for the implementation of a live flexible distribution grid. Beyond that, a more precise analysis showed that, analog to [1], dangerous sign errors in the sensitivity values correspond to the reversal of $\mathrm{P}$ or $\mathrm{Q}$ flow at the respective line or transformer and therefore to a very low sensitivity value. This low value excludes the consideration in a market from the outset because high sensitivity values are preferred due to efficiency and economic reasons.
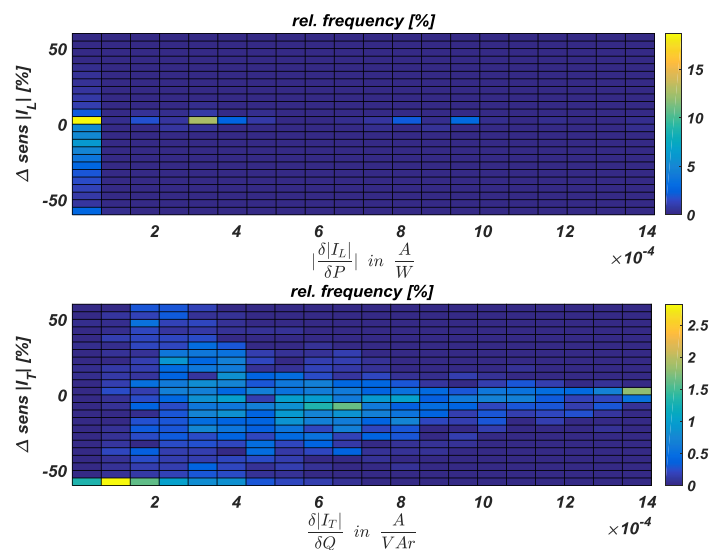

Figure 5: Correlation between sensitivity values and error for all values within a simulation period of two weeks.

\section{FIELD TEST RESULTS}

There are some deviations in the field test compared to the simulations. Due to the fact that the number of connected costumers in the investigated grid is rather small, complete symmetrical system operation is not common. In addition to this, all battery storages are connected to Phase B in order to provoke distinct and measurable reactions in the grid increasing the degree of unbalance. Moreover, the sporadic failure of data transmission leads to a limited availability of the measurement values and a reduced amount of input data for the state estimation. The behaviour of the batteries presented in this paper is not motivated by solving grid problems as it is common in a flexible distribution grid but rather to provide a foundation for a sensitivity evaluation.
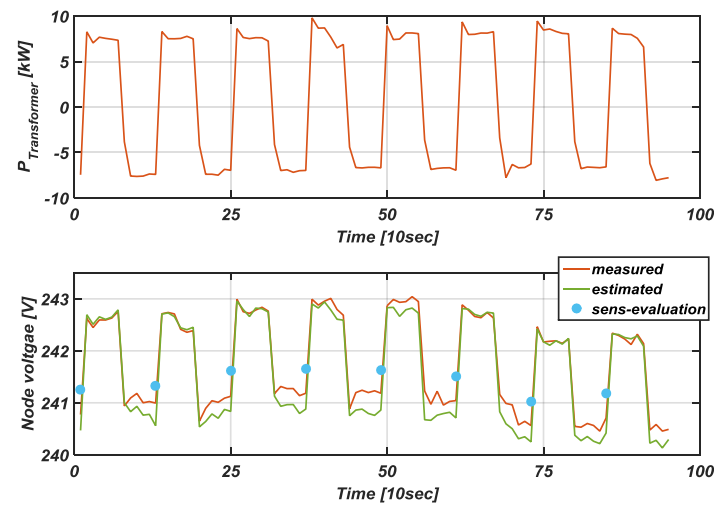

Figure 6: Power flow across the transformer (above) and real, estimated and with the help of sensitivity values calculated voltage at node i (below) over a few minutes. 
Nevertheless, for simplicity reasons a symmetrical state estimation combined with a slightly adjusted symmetrical sensitivity analysis was applied to the values of Phase B. Slightly adjusted means that the values are multiplied by the factor three due to the threefold power over Phase $\mathrm{B}$ in comparison to a symmetrical consideration. The accuracy of the sensitivity values calculated under these circumstances was then analyzed. With the objective to alternate the voltage level in the grid all battery storages switch their power between maximal feed in $(3.7 \mathrm{~kW}$ each) and zero during night hours as it can be seen by the alternating transformer power in figure 6. In the lower part of figure 6 , the measured voltage at a representative node is displayed alongside the estimated voltage, indicating an acceptable concurrence. The predicted voltage value for the time point $t+1$ according to the sensitivity values at time point $t$ was calculated as shown below.

$$
V_{t+1}=V_{t}+\sum_{i=1}^{4} \frac{\delta V}{\delta P_{i}} \cdot \Delta P_{i}, \text { with } \Delta P_{i} \approx 3,7 \mathrm{~kW}
$$

The blue dots in figure 6 show the result of the prognosis which was conducted every $60 \mathrm{sec}$, directly before a feed in period. Due to the fact that other power flow modifications can be neglected during that time, the bad results can be attributed to the negligence of unsymmetrical conditions. Furthermore, also the state estimation results show a bad accuracy for other nodes (figure 7) occasionally leading to convergence problems with an increasing degree of unbalance.

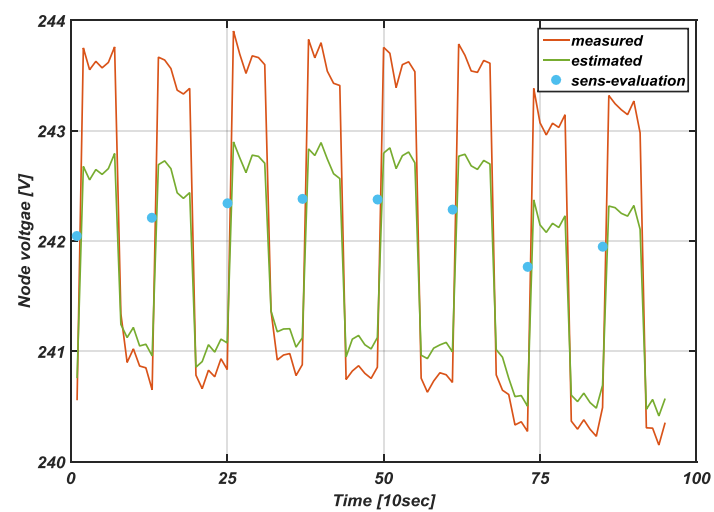

Figure 7: Real, estimated and with the help of sensitivity values calculated voltage at node $\mathrm{k}$ over a few minutes with a low accordance between measured and estimated voltage

\section{CONCLUSION AND OUTLOOK}

Referring to the simulation results, the investigated errors for both state estimation and sensitivity were within an acceptable range for the application within a live flexible distribution grid. However in the field test a symmetrical investigation (regarding Phase $\mathrm{B}$ and assuming that Phase A and B behave identical) leads to high errors. It could be seen that the real sensitivities exhibit distinctly higher values in comparison to the calculated symmetrical ones (factor six would be a better approach than three as it rudimentarily includes the neutral conductor). Due to this, a detailed unsymmetrical investigation is recommended which could be a problem for most of the low voltage grids. In several cases it is not known at which phase a certain potential flexibility option is connected, leading to a non-applicability in a flexible distribution grid. However the requirement of a sufficient number of competitors (connected at the same phase) must be met.

\section{ACKNOWLEDGEMENT}

The work presented in this paper is funded by the German Federal Ministry of Economic Affairs and Energy (BMWi) within the project In2VPP (contract number: 0325607). The In2VPP project is a cooperative effort of infra fürth gmbh, OFFIS e.V., Siemens AG and Technical University of Munich (TUM) with the goal of bridging the gap between the economic and technical challenges associated with VPPs.

\section{REFERENCES}

[1] M. Wagler, R. Witzmann, 2016, "Sensitivity Analysis - A Key Element for the Operation of a Flexible Distribution Grid", IEEE PES Transmission and Distribution Conference, Dallas

[2] C. Glomb et al., 2016, "Grid-Aware VPP Operation", IEEE International Energy Conference Energycon, Leuven

[3] A. Gjelsvik, S. Aam, L. Holten 1985, "Hatchel's augmented matrix method - a rapid method improving numerical stability in power system state estimation", IEEE Transaction on Power Apparatus and Systems

[4] D. Waeresch et al., 2016, "State Estimation in low voltage grids based on smart meter data and photovoltaic-feed-in forecast", $23^{\text {th }}$ Conference on Electricity Distribution CIRED, Lyon

[5] A. Abur, A.G. Expósito, 2004, Power System State Estimation, CRC Press, Boca Raton, USA

[6] W. Peres et al., 2014, "Branch current based state estimation: Equality-constrained WLS and Augmented Matrix Approaches", Congresso rasileiro de Automatica, Belo Horizonte 